\title{
El manuscrito inédito de La Florida del Inca (1605) en las manos del cronista real de Indias Antonio de Herrera
}

\section{The Unpublished Manuscript of The Florida of the Inca (1605) in the Hands of the Royal Chronicler of Indies Antonio de Herrera}

\section{Andrea V. López}

Dickinson College

ESTADOS UNIDOS

lopezand@dickinson.edu

[Hipogrifo, (issn: 2328-1308), 9.2, 2021, pp. 653-674]

Recibido: 27-01-2021 / Aceptado: 23-02-2021

DOI: http://dx.doi.org/10.13035/H.2021.09.02.48

Resumen. El presente artículo estudia el manuscrito de La Florida del Inca publicado por Miguel Maticorena Estrada con el título «La Florida»: facsímil de un nuevo manuscrito (2015). El objetivo del estudio es corregir el estado de la crítica actual que establece que este texto fue una versión preliminar de La Florida del Inca (1605) del Inca Garcilaso de la Vega. Esta investigación sustenta que el manuscrito de Maticorena constituye un documento de trabajo elaborado por el cronista mayor de Indias Antonio de Herrera y Tordesillas durante su tarea de síntesis y adaptación de la obra del Inca para insertarla en la crónica oficial española. El estudio se divide en tres secciones. En primer lugar, se indaga en la vía burocrática por la cual Antonio de Herrera obtuvo el manuscrito inédito de La Florida del Inca que utilizó para elaborar el manuscrito encontrado por Maticorena. En segundo lugar, se realiza un cotejo entre la caligrafía presente en el manuscrito de Maticorena y algunos documentos autógrafos de Antonio de Herrera que confirma su autoría. En tercer lugar, se analizan algunos elementos del manuscrito que revelan aspectos centrales del método historiográfico de Antonio de Herrera. 
Palabras clave. Inca Garcilaso de la Vega; La Florida del Inca; Antonio de Herrera y Tordesillas; La Florida española.

Abstract. This article studies the manuscript of La Florida del Inca (1605) discovered and published by Miguel Maticorena Estrada with the title of «La Florida»: facsímil de un nuevo manuscrito (2015). I discuss the current state of the criticism which establishes that the manuscript was a preliminary version of La Florida del Inca by Inca Garcilaso de la Vega. I aim to prove that Maticorena's manuscript constitutes a summary of a completed version of La Florida del Inca created by the Spanish chronicler of Indies Antonio de Herrera y Tordesillas. Moreover, I argue that the manuscript was Herrera's work document created with the objective of inserting Garcilaso's work into the official Spanish chronicle. The article is divided into three sections. First, I examine the bureaucratic route by which Antonio de Herrera obtained the unpublished manuscript of La Florida del Inca that he employed to create the manuscript discovered by Maticorena. Second, I present a calligraphic comparison between Maticorena's manuscript and Antonio de Herrera's personal documents that confirm its authorship. Third, I analyze specific elements of the manuscript that reveal central aspects of Antonio de Herrera's methodology of writing history.

Keywords. Inca Garcilaso de la Vega; La Florida del Inca; Antonio de Herrera y Tordesillas, Spanish La Florida.

Antes de la publicación de La Florida del Inca (1605), el Inca Garcilaso había alcanzado reconocimiento dentro de los círculos intelectuales de su tiempo por su traducción del complejo tratado neoplatónico Dialoghi d' Amore del poeta judío Judá Abravanel -conocido por su nombre hispano León Hebreo- titulada Tradución del indio de los tres Diálogos de amor de León Hebreo (1590). En la dedicatoria de su obra a Maximiliano de Austria, abad de Alcalá la Real, Garcilaso menciona a varios intelectuales que respaldan la calidad de su obra ${ }^{1}$. Mediante las referencias a estas destacadas personalidades, Garcilaso buscaba presentarse como miembro del círculo intelectual de su época.

Los paratextos de La Florida del Inca, por el contrario, carecen de nombres relacionados al ámbito académico. Entre los nombres silenciados destaca su fuente principal, después identificada como Gonzalo Silvestre ${ }^{2}$. Garcilaso solo menciona sus dos fuentes secundarias, los escritos de Alonso de Carmona y Juan Coles, que

1. Entre ellos están Agustín de Herrera, maestro en Teología y preceptor de don Pedro Fernández de Córdova, marqués de Priego; Jerónimo de Prado, jesuita y exégeta; Pedro Sánchez de Herrera, licenciado y teólogo montillano; y Fernando de Zárate, agustino y catedrático jubilado de la universidad de Osuna. El Inca agrega que se abstiene de mencionar muchos otros nombres de "personas graves" para no volver tediosa la lectura «y otros religiosos y personas graves que por no cansar a VS. no las nombro». Garcilaso, Traduzión del Indio de los tres diálogos de amor de León Hebreo, p. 15.

2. José de la Riva-Agüero estableció la identidad del principal informante del Inca en La historia en el Perú, 1910 (Riva-Agüero, 1965); lo cual fue luego comprobado por José Durand en «La redacción de La Florida del Inca: cronología» (1962), con amplia documentación que registró las visitas de Garcilaso al pueblo de Las Posadas, residencia de Gonzalo Silvestre. 
enriquecieron su primera redacción de La Florida basada en el relato de Silvestre. El Inca tampoco menciona el nombre del cronista real que corroboró la veracidad de su narración contrastándola con la documentación oficial del Consejo de Indias. Este cronista real fue luego identificado como Ambrosio de Morales, a quien el Inca solía enviar los manuscritos inéditos de su obra ${ }^{3}$. El único historiador de las Indias que Garcilaso menciona en su dedicatoria de La Florida es fray Pedro de Aguado (1538-1589), un franciscano comisionado a Santa Fe en Nueva Granada. El Inca señala que Juan Coles compuso su breve relación sobre los sucesos de La Florida a petición de Aguado que en aquella época era provincial de Santa Fe 4 .

La ausencia de referencias a otros intelectuales en los paratextos de La Florida revela un cambio en la auto-representación del historiador mestizo. El Inca se aleja de la postura que construye su autoridad sobre un colectivo intelectual y eclesiástico; en su lugar, adopta una posición de mayor autonomía que busca darle solución a una problemática de estado. Garcilaso constantemente declara que el objetivo central de La Florida del Inca es que España logre efectuar exitosamente la conquista del territorio del mismo nombre: «para que hoy más [...] se esfuerce España a la ganar y poblar... pretendiendo yo incitar y persuadir con la relación de esta historia a que los españoles ganen aquella tierra para aumento de nuestra Sancta Fe Católica» ${ }^{5}$. Es decir, La Florida tiene una finalidad pragmática dentro de un contexto geopolítico conflictivo. Posiblemente, este aspecto fue determinante para que Antonio de Herrera y Tordesillas, cronista oficial de la Corona española, recogiera e insertara la obra de Garcilaso en la crónica oficial española.

Durante el periodo en que Garcilaso escribió La Florida del Inca y cuando Antonio de Herrera escribió su Historia general de los hechos de los castellanos en Tierra Firme y mar Océano (1601-1616) conocida como Décadas, La Florida era el territorio americano más importante en disputa entre los imperios europeos. Francia, España y, posteriormente, Inglaterra establecieron colonias y se enfrentaron por la posesión del territorio floridiano ${ }^{6}$. En la problemática situación geopolítica de La Florida, Garcilaso encontró la oportunidad para proponer un modelo de colonización basado en la reivindicación de los líderes nativos americanos. Sobre La Florida del Inca se ha escrito que Garcilaso se dedica a construir un nuevo imaginario del indígena americano que luego va a reafirmar y continuar en los Comen-

3. Una carta del Inca con fecha de 31 de diciembre de 1592 publicada por Eugenio Asensio comprueba la cercanía entre Garcilaso y Ambrosio de Morales. En ella Garcilaso dice afectuosamente de Morales que «me adoptó por hijo y tomó por suyos mis trabajos» (Asensio, 1953, p. 588).

4. La Real Academia de la Historia (en la ficha «Aguado, Pedro de») refiere que Aguado fue elegido provincial en 1573, año en que iniciaría su obra historiográfica. Aguado tituló su obra Recopilación historial, donde trató los eventos del descubrimiento, conquista y colonización de la región norandina de Nueva Granada.

5. Inca Garcilaso de la Vega, La Florida del Inca, pp. 5-7.

6. El francés y líder protestante René Goulaine de Laudonnière fundó el primer asentamiento permanente en La Florida, Charles Fort, en 1564. Posteriormente, Pedro Menéndez de Avilés expulsó a los franceses y fundó la ciudad de San Agustín en 1565. Finalmente, la compañía inglesa London Company patrocinó la fundación de la colonia Jamestown en 1607. Ver Dewhurst, 1968; Hoffman, 1999; Mc Grath, 2000; Tebeau, 1971 
tarios reales ${ }^{7}$. Sin embargo, no se ha considerado que el objetivo central de esta representación fue influenciar las políticas colonizadoras de su tiempo a favor de la nobleza nativa.

Así, con La Florida del Inca, Garcilaso comenzó a participar públicamente en el debate acerca de las capacidades de los indígenas americanos. Este debate se nutría de la abundante producción escrita de historiadores, teólogos, juristas y otros intelectuales de la época que discutían la legitimidad del dominio de los indígenas americanos. Para ingresar a la conversación, Garcilaso tuvo que ajustar su contribución a los requerimientos que imponía la época. Es decir, tuvo que someter su obra al complejo proceso de publicación establecido por el brazo burocrático de la Corona española y la Iglesia católica ${ }^{8}$. Finalmente, este proceso ocasionó que el manuscrito inédito de La Florida del Inca llegue a manos del cronista mayor de Indias, como se detallará más adelante.

Sobre estas bases, el presente artículo aborda una serie de cuestionamientos que emergen de la pugna invisible de Garcilaso por entrar al ámbito público como historiador. El camino transitado por el manuscrito inédito de La Florida del Inca se puede reconocer a partir del proceso de apropiación que Antonio de Herrera ejerció sobre él. Para reconstruir el tratamiento que Herrera le da al texto de Garcilaso, analizaré las características materiales del manuscrito de La Florida del Inca encontrado y publicado por Miguel Maticorena en 2015, para así determinar la identidad del autor de esta versión manuscrita9 ${ }^{9}$. Demostraré que este manuscrito contiene una versión abreviada de la crónica de Garcilaso que erróneamente ha sido catalogada por la crítica como un borrador del Inca, hecho que ha impedido el estudio apropiado de su contenido.

El presente artículo corrige este estado de la crítica y prueba que el manuscrito publicado por Maticorena no fue un borrador de Garcilaso, sino un documento de trabajo elaborado de propia mano por el cronista real Antonio de Herrera durante su tarea de apropiación y edición de la crónica del Inca ${ }^{10}$. En síntesis, este artículo busca responder a las siguientes preguntas: ¿Cuál fue el marco institucional al que tuvo que someterse Garcilaso para que su obra alcanzara el ámbito público, es decir, la publicación? ¿Cuáles fueron los medios por los que Antonio de Herrera logró

7. Serna Arnaiz, 2008, p. 129.

8. El arribo de la imprenta al imperio español se produjo un poco más tarde que en otros imperios. Sin embargo, la pragmática Real de 1480 instaurada por Isabel la Católica permitiendo la circulación de libros extranjeros y liberándolos de los derechos de alcabalas generó el rápido crecimiento de la imprenta. Ver Torre Revello, 1940, p. 33. Más tarde, la legislación fue restringiendo estas libertades con las sucesivas pragmáticas reales que condicionaron la publicación de los libros bajo un complejo proceso burocrático a cargo del Consejo de Indias, el Consejo de Castilla y las instancias religiosas pertinentes. 9. Esta edición facsímil lleva como título La Florida, facsímil de un nuevo manuscrito y fue publicado por la Universidad Ricardo Palma en 2015.

10. Recientemente, José Miguel Martínez Torrejón (2020) ha publicado una edición crítica del manuscrito encontrado por Maticorena. En su estudio del manuscrito, Martínez también arriba a la conclusión de que Antonio de Herrera es el autor autógrafo del manuscrito. 
acceder a la versión inédita de La Florida del Inca? ¿Cuáles son las características específicas del manuscrito encontrado por Maticorena que revelan la identidad de su autor?

Demostraré que Antonio de Herrera utilizó los privilegios de su cargo de cronista real para acceder a la versión inédita de La Florida del Inca. Después de obtenerla, con el propósito de integrar la información provista por Garcilaso a su proyecto de escritura, Herrera realizó un cuidadoso proceso de selección, síntesis y reformulación de los eventos narrados en La Florida del Inca. Utilizando muestras caligráficas de Herrera, probaré que el manuscrito encontrado por Maticorena, en adelante $M$, es una síntesis elaborada por el cronista real de una versión inédita de La Florida. En consecuencia, lejos de ser el borrador de La Florida del Inca de Garcilaso, el manuscrito M fue una versión preliminar de las Décadas de Antonio de Herrera. Este descubrimiento permite estudiar los criterios de edición y manipulación de Herrera sobre la obra de Garcilaso y desmiente la teoría de que Garcilaso utilizó el manuscrito M como base para un proceso de invención retórica que culminaría en su publicación de 1605.

En la primera sección de este artículo describiré los medios empleados por Antonio de Herrera para obtener acceso a la obra del Inca. En la segunda sección, cotejaré algunas grafías del manuscrito $\mathrm{M}$ con documentos personales de Herrera que corroboran la autoría del cronista real. En la tercera sección me enfocaré en las fracturas de transcripción presentes en el manuscrito. Seguiré las enmiendas para establecer algunos lineamientos de la metodología de escritura de Herrera, sus criterios de edición y las filtraciones de la retórica de Garcilaso que pasaron al manuscrito M. Estos elementos demuestran que la sección de las Décadas dedicada a la expedición de Hernando de Soto es el producto de un meticuloso proceso de síntesis y reformulación de información que Herrera inició con la lectura de una versión previa e inédita de la Florida del Inca.

\section{LA CIRCULACIÓN DEL MANUSCRITO DE LA FLORIDA DEL INCA}

Antes de la impresión de La Florida del Inca en 1605, ya existían tres crónicas sobre la expedición liderada por Hernando de Soto. Dos de ellas circularon públicamente y una se mantuvo en los archivos reales. Estas son: los diarios de Rodrigo Rangel, secretario de De Soto, que Gonzalo Fernández de Oviedo reprodujo en su Historia general y natural de las Indias, la cual no se publicó pero se conservó en los archivos reales; la Relación del suceso de la jornada que hizo Hernando de Soto y de la calidad de la tierra por donde anduvo (1544) de Luis Hernández de Biedma, el contador de la expedición; y la Relaçam verdadeira dos trabalhos que o gobernador dom Fernando de Souto e certos fidalgos portugueses passarom no descobrimiento da provincia da Frolida (1557) del Fidalgo de Elvas. Entre las prerrogativas del cargo de cronista mayor de Indias se incluía el acceso a todos estos documentos. Por ello, Antonio de Herrera tenía la potestad de ingresar al archivo de la Corona que en aquel tiempo no estaba abierto para consulta pública. Sin embargo, sus Décadas reflejan que, descartando otras versiones, Herrera siguió los episodios 
según como eran narrados por Garcilaso en La Florida del Inca. Esta preferencia revela que el cronista real encontró en la propuesta de Garcilaso una versión que se ajustaba mejor a los fines de su obra.

La relación entre el manuscrito inédito de Garcilaso y la crónica oficial de España se inició el año de 1596 cuando Antonio de Herrera obtuvo el cargo de cronista mayor de Indias y asumió la responsabilidad de elaborar una crónica general de la conquista del Nuevo Mundo. Rápidamente, Herrera se dispuso a cumplir con esta tarea y cinco años después publicó la primera parte de la Historia general de los hechos de los castellanos en Tierra Firme y mar Océano (1601). Esta primera parte de su obra comprendía el periodo desde la preparación de los viajes de Cristóbal Colón hasta las primeras provisiones reales para el gobierno de Nueva España en 1526.

Retóricamente, el estilo de Herrera corresponde a un tipo de narrativización del modelo de los anales medievales ya que su escritura es extremadamente concisa y está organizada en un estricto orden temporal. Por esta razón aparece frecuentemente fragmentada. Es decir, la narración de un evento se interrumpe para dar paso a sucesos que ocurrieron al mismo tiempo en un espacio geográfico distinto. Así, por ejemplo, la expedición de Hernando de Soto se empieza a narrar en la sección correspondiente al año 1539. Sin embargo, la narración se interrumpe al llegar el año 1540. Allí Herrera inserta la excursión que el conquistador Jorge Robledo dirigió al río Magdalena. Este criterio de organización está presente en los márgenes del manuscrito $\mathrm{M}$ donde Herrera anota los años en que se producen los eventos, probablemente con el propósito de facilitar su posterior incorporación a la versión final de su crónica.

La narración de la expedición de Hernando de Soto inicia en la segunda parte de la crónica de Herrera, Década VI, que se publicó en 1615. Sin embargo, la primera parte impresa en 1601 contiene un detalle que revela el periodo temporal en que Herrera tuvo acceso a La Florida inédita de Garcilaso. En la Década I bajo la «Suma del Privilegio» fechada en 1600, Herrera coloca una lista con los nombres de los cronistas cuyas obras empleó para escribir la suya, entre ellos incluye el nombre «Garcilasso Inga». Bajo esta lista se indica «se han seguido en esta historia los papeles de la cámara real y reales archivos, los libros registros y relaciones y otros papeles del Real y Supremo consejo de Indias, dejando a parte muchas cosas que los autores han dicho por no poderse verificar con escrituras auténticas» ${ }^{17}$. La presencia del nombre de Garcilaso entre las referencias de la primera Década demuestra que en 1601 Antonio de Herrera ya había tenido entre sus manos la versión inédita de La Florida de Garcilaso.

Sin embargo, esta mención entre las fuentes de Herrera no explica la forma en que el cronista real tuvo acceso a la obra de Garcilaso. Para ello es necesario indagar en los procedimientos institucionales que siguió el Inca para alcanzar la publicación de La Florida. Como aspirante a historiador, Garcilaso debía obtener una licencia según las leyes que regulaban la impresión de libros. Para lograr la publicación de sus obras, los autores debían someterlas a un tedioso proceso bu-

11. Herrera y Tordesillas, Historia general, fol. 2r. 
rocrático de calificación establecido por la monarquía y el aparato eclesiástico, un procedimiento del tipo que Mariano José de Larra trescientos años después describiría satíricamente en su artículo Vuelva usted mañana.

La Iglesia Católica instituyó por primera vez en 1521 un sistema de revisión y censura para detener la circulación de textos relacionados a la reforma protestante. Así, los primeros libros en prohibirse fueron los ensayos de Martín Lutero ${ }^{12}$. Felipe II ordenó la primera legislación particular para los libros de temática sobre el Nuevo Mundo en una Real Cédula en 1556 donde mandaba que se recogiesen todos los libros de materia indiana impresos hasta la fecha para que en adelante fueran sometidos a la aprobación del Consejo de Indias para su publicación ${ }^{13}$.

En 1560 una nueva cédula real reiteró la obligación de conseguir la licencia del Consejo de Indias, así como del Consejo de Castilla para la publicación de libros. De acuerdo con Rolena Adorno, esta insistencia revela la inefectividad de la legislación. Es decir, frente a las ordenanzas de la Corona, los impresores mantenían una actitud de «obedezco, pero no cumplo» ${ }^{14}$. José Torre Revello en su estudio sobre la publicación de libros de temas americanos en España ya había admitido que «a pesar de las leyes promulgadas y de las medidas tomadas por las autoridades, no se daba mayor cumplimiento a parte de lo ordenado, y los impresores daban a luz toda clase de libros» ${ }^{15}$. El Inca Garcilaso, no obstante, durante su actividad como escritor siempre procuró cumplir todos los requerimientos oficiales para la publicación de su obra. Esa actitud, paradójicamente, terminaría exponiendo La Florida del Inca a la manipulación de Herrera.

Garcilaso no contaba con ningún tipo de apoyo interno en el aparato administrativo, por lo que estos procedimientos complicaron el destino de La Florida. La Tradución, que había gozado del mecenazgo de Maximiliano de Austria, obtuvo la licencia sin mayores retrasos y se publicó en la importante imprenta de Pedro Madrigal en Madrid ${ }^{16}$. Sin embargo, cuando Garcilaso llevó su manuscrito de La Florida al Consejo de Indias para someterlo a revisión y obtener la licencia de impresión, se encontró con la intervención de Antonio de Herrera y Tordesillas. Herrera aprovecharía la posición de su cargo para acceder al trabajo inédito de Garcilaso e insertarlo posteriormente en la crónica oficial de España, lo cual resultó determinante en el destino de la obra del Inca.

12. Benito Arias Montano, el director del proyecto editorial que produjo la Biblia Políglota de Amberes creó el primer índex de libros examinados y expurgados por la Iglesia Católica en 1570. Ver Adorno, 2020, p. 30

13. «R. C. a las autoridades de Castilla, ordenándoles, que no se permita imprimir ningún libro sobre materias del Nuevo Mundo, sin que antes lo examine el Consejo de Indias y que se recojan los que se hubiesen impreso sin ese requisito» (Torre Revello, 1940, p. XII).

14. Adorno, 2020, p. 51

15. Torre Revello, 1940, p. 28.

16. La imprenta fundada por Pedro Madrigal fue una de las principales imprentas madrileñas del siglo XVI. Las publicaciones de la imprenta de Madrigal incluyeron obras en castellano y latín, entre las que predominaban importantes tratados de materia legislativa y teológica. «Madrigal, Pedro». 
Como se ha mencionado líneas arriba, en 1556 la Corona ordenó que el Consejo de Indias revisara los libros de materia indiana. En 1558, Felipe II expidió una Real Pragmática por la que prohibía la circulación de cualquier libro impreso sin licencia del rey y de su Consejo de Castilla. Para alcanzar la ansiada licencia, el autor debía presentar el manuscrito de la obra, después de censurado, al escribano de Cámara del Consejo. El escribano debía rubricar y numerar todas sus páginas. Luego de lo cual se colocaban en hojas aparte las erratas, para que se corrigiera o se suprimiera esa parte de los originales. Una vez enmendado y censurado el manuscrito de una obra se remitía a la imprenta. Efectuada la impresión, debía devolverse al Consejo uno o dos ejemplares para confrontarlos con el manuscrito original. Una vez obtenida la conformidad de la impresión con el manuscrito, se le otorgaba al autor la licencia, la tasa de venta y la cédula de privilegio. El manuscrito original se debía conservar en los archivos del Consejo. Asimismo, se colocaba en un registro el título de la obra, las licencias otorgadas y los nombres del autor y de los revisores ${ }^{17}$.

Este engorroso proceso fue la causa de que muchos impresores desatendieran lo señalado por las pragmáticas, por lo que «poco o nada les importaba editar uno, y otro, y muchos libros sin aprobación, tasa, privilegio, ni aun licencia» ${ }^{18}$. El mismo protocolo era aplicado por el Consejo de Indias para la evaluación de las obras remitidas a él. En 1599 Garcilaso decidió pedir la licencia de publicación para La Florida al Consejo de Indias. Con este propósito, le otorgó un poder a Juan de Morales, portero del Consejo ${ }^{19}$. A pesar de que Morales obtuvo la licencia, por razones aún desconocidas la publicación no se realizó. José Durand especula, y todas las pruebas parecen darle la razón, que la intervención de Antonio de Herrera estuvo detrás del retraso en la publicación de La Florida: «Creo cierto que Herrera, autor de conocidas fechorías, retardaba la publicación para amparar el plagio e impedir futura competencia» ${ }^{20}$. Cinco años después, en 1604, el Inca otorgó otro poder a Domingo de Silva, nuevamente con el objetivo de obtener la licencia, pero esta vez ante el Consejo de Castilla ${ }^{21}$. Al año siguiente La Florida se publicó en Lisboa con dedicatoria al duque Teodosio de Braganza, aspirante al trono portugués.

De este escenario podemos obtener algunas conclusiones. En primer lugar, el Consejo de Indias fue el vínculo entre La Florida inédita y Antonio de Herrera. El cronista mayor dispuso de todos los documentos entregados al Consejo, entre ellos el manuscrito de Garcilaso, como lo indica la nota bibliográfica bajo el «Privilegio» de la Década I. Además, dentro del protocolo de evaluación se establecía que el manuscrito original se debía conservar en el archivo del Consejo, lo cual facilitaba su acceso. El manuscrito M tiene una anotación final que hace referencia a uno de los episodios dentro de la crónica: «la prueba de las cotas que pasaban las flechas dice Garcilaso a hojas 192 y se pusieron en un cesto» ${ }^{22}$. La anotación prueba que

17. Torre Revello, 1940, pp. 26-27.

18. Pérez Pastor, 1895, p. X.

19. Torre y del Cerro, 1935, pp. 33-37.

20. Durand, 1990, p. 6.

21. Torre y del Cerro, 1935 , pp. 53-55, 60-61 y 104-106.

22. Maticorena Estrada, 2015, p. 263. En todas las citas modernizo las grafías sin relevancia fonética. 
Herrera utilizó una versión numerada de la crónica de Garcilaso para escribir el manuscrito M. Puede tratarse del manuscrito numerado por el escribano del Consejo que se conservaba en el archivo o de la prueba de impresión que era cotejada con el manuscrito original.

En segundo lugar, el acceso del cronista mayor a La Florida inédita fue casi inmediato. Garcilaso envió La Florida al Consejo de Indias para su aprobación en 1599 y solo dos años después, en 1601, Antonio de Herrera registra su nombre entre las referencias bibliográficas de las Décadas. La inclusión de Garcilaso en la lista de fuentes demuestra que Herrera tenía un gran interés por la obra del Inca y estaba decidido a incorporarla en su crónica. Por ello, lo menciona a pesar de que la expedición de Hernando de Soto no aparece en la primera parte de las Décadas.

Por último, se deduce que Garcilaso tuvo una gran urgencia por publicar su obra y que sospechaba que su crónica podía ser plagiada. Esta deducción se desprende de ciertas secciones de su obra en que se refieren al hurto literario, una de las cuales alude directamente al caso de La Florida. El Inca proyecta este temor en la semblanza de su pariente, el poeta Garcí Sánchez de Badajoz en la Relación de la descendencia del famoso Garcí-Pérez de Vargas (1596), donde comenta que los autores no deben desamparar sus obras inéditas ya que se exponen al plagio: «que otros que no merescen ser discípulos ni aun criados del único Garcí Sánchez de Badajoz, por ver esta su obra vedada y desamparada la hurten a pedazos para ilustrar sus poesías engastándolas en ellas como yo los he visto en las de algunos poetas hechos famosos y ricos con tesoro ajeno» ${ }^{23}$.

De forma similar, en la primera parte de los Comentarios reales (1609) Garcilaso declara su sospecha de que La Florida había sido plagiada: «Lo que ahora temo es que no me las haya hurtado algún historiador, porque aquel libro [La Florida] por mi ocupación, fue sin mí a pedir su calificación, y sé que anduvo por muchas manos» ${ }^{24}$. A pesar de que el Inca no señala al cronista real, parece que Herrera se sintió aludido ya que seis años después, cuando publicó su Década VI, se esforzó por borrar todo indicio de la autoría de Garcilaso. Por ello, afirmó dos veces que su fuente de información fue la relación que un fraile menor llevó al Consejo de Indias. Primero indica «que la traía de México, adonde la escribió uno de los Capitanes que se hallaba en esta jornada» ${ }^{25}$. Luego, insiste al final de la narración diciendo «llegó esta historia a manos del Obispo de Córdoba don Pablo de Laguna, la cual siendo presidente del Supremo Consejo de las Indias le dio un fraile menor (como se ha dicho)» ${ }^{26}$. Este intento por eliminar los rastros de la autoría de Garcilaso se reflejó en la reformulación de los eventos de la expedición en los cuales Herrera procuró eliminar las huellas de la retórica del Inca, aunque sin lograrlo completamente, como se verá adelante. Pero antes, es necesario descender al nivel más cercano a la elaboración textual de Herrera y Tordesillas.

23. Inca Garcilaso de la Vega, Relación de la descendencia del famoso Garcí-Pérez de Vargas con algunos pasos de historias dignas de memoria, fol. $4 \mathrm{v}$.

24. Inca Garcilaso de la Vega, Comentarios reales de los Incas, p. 17

25. Herrera, Historia general, p. 29.

26. Herrera, Historia general, p. 185.

HIPOGRIFO, 9.2, 2021 (pp. 653-674) 


\section{LA MANO DE ANTONIO DE HERRERA}

El año 2015 el fondo editorial de la Universidad Ricardo Palma publicó en edición facsímil un manuscrito encontrado por el reconocido garcilasista peruano Miguel Maticorena Estrada con el título «La Florida»: facsímil de un nuevo manuscrito. Este documento que comprende cuarenta folios da cuenta de los sucesos ocurridos en la expedición dirigida por Hernando de Soto y finaliza casi con su muerte. En el prólogo y en el estudio introductorio del facsímil, Eduardo Hopkins y Miguel Maticorena sostienen que el manuscrito M fue la narración dictada por Gonzalo Silvestre, el principal informante de Garcilaso, durante sus entrevistas en Las Posadas entre 1587 y 158927 . Según esta hipótesis, M contiene la materia prima que luego Garcilaso reformuló y amplificó para componer La Florida del Inca. Es decir, ambos críticos postularon que $\mathrm{M}$ fue el borrador que sirvió de base para la invención retórica de Garcilaso.

Según Maticorena, un cotejo entre M y la versión final de La Florida del Inca, revela los elementos más originales de la elaboración literaria del Inca Garcilaso: «El cotejo del manuscrito con la edición [La Florida] deja ver el trabajo artístico del Inca» ${ }^{28}$. Asimismo, Hopkins señala que la retórica de Garcilaso en La Florida se diferencia del manuscrito M por su intención ejemplarizante y el carácter universalizador que le brinda a la historia: «Garcilaso construye juicios sobre los hechos históricos, con el fin de realizar proyecciones hacia el presente y el futuro» ${ }^{29}$. También agrega que la visión ejemplarizante de la obra se condensa en las intervenciones del narrador que en ocasiones interpreta o censura las acciones de los conquistadores. Sin embargo, la ausencia de la ejemplaridad y de los episodios de carácter universalizador no revelan la elaboración retórica de Garcilaso, sino el estilo historial de Antonio de Herrera, quien seleccionó y recortó los episodios de La Florida del Inca. Es decir, los elementos que diferencian a M de La Florida responden al estilo y los objetivos de Herrera y no a los de Garcilaso.

Maticorena también plantea que Herrera tuvo acceso al manuscrito M y que reprodujo casi íntegramente todos los eventos registrados en él. Sin embargo, no reconoce que la caligrafía del cuerpo del manuscrito y de las anotaciones que están en los márgenes y al final del documento son del cronista real. Así, Maticorena propone dos líneas de desarrollo textual independientes a partir de M. Por un lado, coloca la versión reproducida casi a la letra por Antonio de Herrera; y por otro, la

elaboración literaria de la mano del Inca Garcilaso.

27. Aurelio Miro Quesada establece esta fecha para las entrevistas entre el Inca y Silvestre: «Consta que allí [Las Posadas] fue a verlo Garcilaso en 1587-1589. Fue en realidad una decisión muy oportuna, porque Gonzalo Silvestre, achacoso por las viejas heridas y la edad, falleció en el verano de 1592» (Miró Quesada, 1956, p. XXII).

28. Maticorena Estrada, 2015, p. 40

29. Hopkins, 2015, p. 14. 
Maticorena identifica tres tipos diferentes de caligrafía en M. En primer lugar, la que presenta el cuerpo del texto: «el texto principal es de una sola mano» ${ }^{30}$. En segundo, las anotaciones al margen; las cuales, especula, pueden pertenecer a Herrera: «Hay varias notas marginales de otra mano. Al margen viene escrito con número los años antes mencionados. Parece que estas fechas proceden del Cronista Mayor Antonio de Herrera» ${ }^{37}$. Por último, se refiere a las anotaciones al final y al principio del manuscrito, las distingue de las otras, aunque también menciona la posibilidad de que pertenezcan a Herrera:

Al final, vienen anotaciones [...] posiblemente de Antonio de Herrera o de alguien interesado en temas militares. Estas notas adicionales, con letra distinta, repetimos, no alteran el texto original [...]. En la primera página y debajo del título viene esta anotación de mano distinta: «Ojo. Costóme mi dinero y no es del Rey». Esto revela que es persona interesada en asuntos de La Florida. Es persona que tiene acceso a papeles oficiales o de Palacio. Podría ser Antonio de Herrera ${ }^{32}$.

En lo último Maticorena no se encontraba lejos de la verdad, pues las grafías presentes en las anotaciones al margen, tanto los años como las adendas iniciales y finales, pertenecen efectivamente al cronista mayor de Indias. Sin embargo, también es de su mano el cuerpo íntegro del texto. En lo que sigue cotejaré las grafías en $\mathrm{M}$ y en unas cartas autógrafas que escribió Antonio de Herrera al arzobispo de Granada, Pedro Vaca de Castro, para probar que ambos documentos fueron elaborados por la misma mano. Presentaré ejemplos suficientes que verifican la identidad entre las letras y las palabras en $\mathrm{M}$, incluyendo las anotaciones al inicio, final, cuerpo y márgenes, y la letra de Herrera en sus cartas privadas y firmadas de su propia mano.

Una de las palabras más utilizadas en los textos referidos es Rey. En la imagen 1 se encuentra esta palabra señalada dentro de una carta que Herrera le escribió a Vaca de Castro agradeciéndole por haberle enviado una relación sobre sucesos en el Perú fechada el 18 de octubre de 1605. En este fragmento se lee: «El Visorrey entró En el Perú, adonde se mostró su verdadera fidelidad en los vaivenes que le dieron los inquietos, prudencia en gobernar las cosas del Rey».

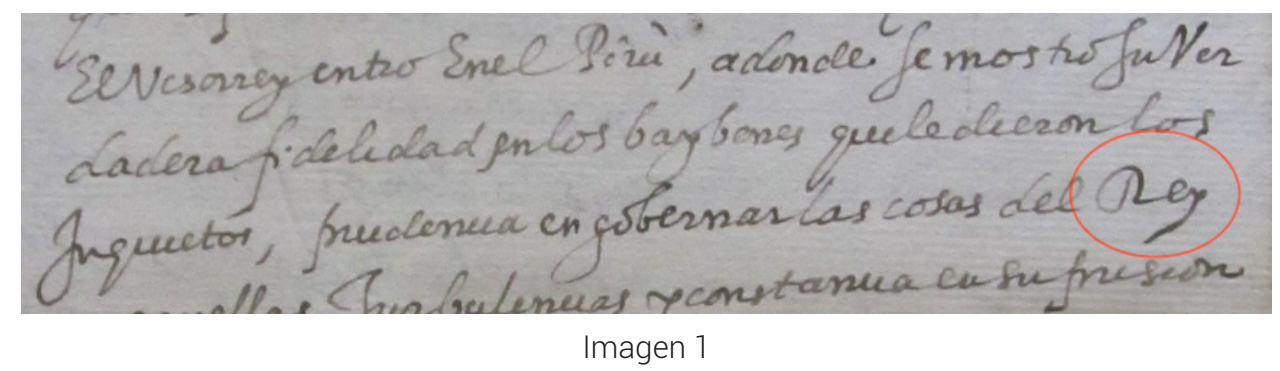

30. Maticorena Estrada, 2015, p. 33

31. Maticorena Estrada, 2015, p. 33

32. Marticorena Estrada, 2015, p. 34 
A continuación, presento dos muestras de las grafías de la palabra Rey en M. La primera corresponde a la anotación debajo del título del manuscrito [imagen 2] y la segunda al cuerpo del documento [imagen 3] ${ }^{33}$.
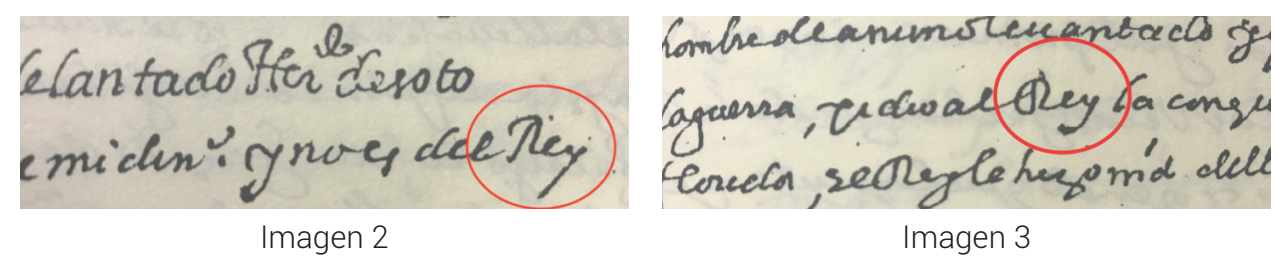

Las tres grafías muestran el mismo patrón. La construcción de la $R$ comienza con la asta del cual se dibuja el bucle sin romper el trazo. La única diferencia clara entre las mayúsculas $R$ se encuentra en la anotación [imagen 2] donde se observa que se realizaron dos trazos: uno para la asta y otro para el bucle de la $R$. Sin embargo, esta doble costumbre para escribir la mayúscula era común en la letra de Herrera, como se comprueba en el siguiente fragmento [imagen 4] de la misma carta al obispo Castro donde se lee: «Receví la Relación» y se observan los dos tipos de escritura de la mayúscula.

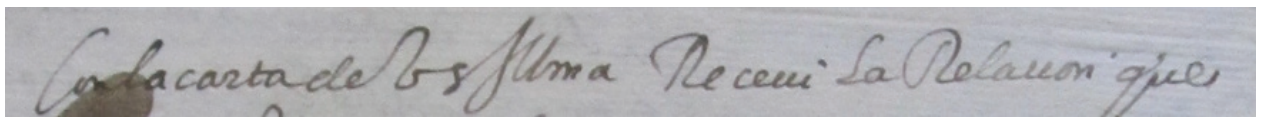

Imagen 4

Otra mayúscula importante para la identificación de la caligrafía de Herrera en el manuscrito $\mathrm{M}$ es la letra $\mathrm{H}$. Los ejemplos que presento a continuación provienen de la firma de Herrera en la carta [imagen 5] y del título del manuscrito M [imagen 6] ${ }^{34}$. La importancia de cada una dentro de su contexto facilita la comparación ya que ambas se escribieron con mayor cuidado que el resto del texto.

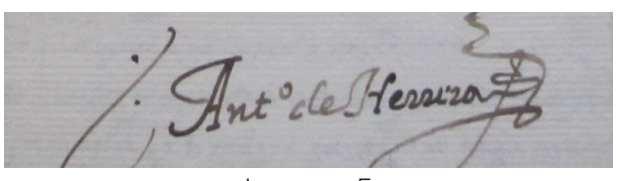

Imagen 5

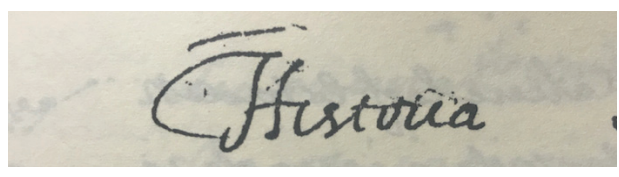

Imagen 6

Ambas mayúsculas se inclinan hacia la derecha y muestran un ligero gancho en las bases y en las astas de la letra. Para comprobar que las anotaciones al margen también son de autoría de Herrera presento, a continuación, dos muestras de la palabra tiempo. Una fue extraída de una carta de Herrera [imagen 7]; la otra, de una de las notas al margen de $\mathrm{M}$ [imagen 8$]^{35}$. 


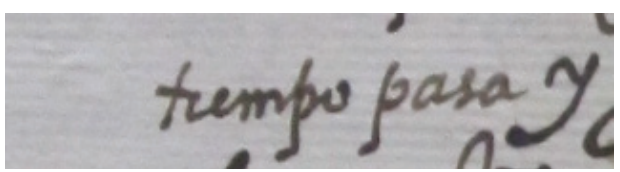

Imagen 7

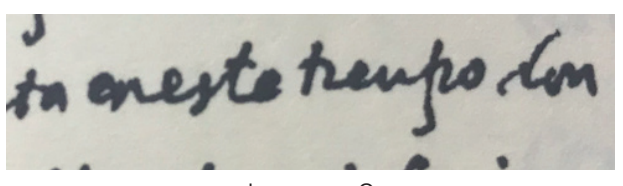

Imagen 8

Ambas muestran el mismo trazo en la composición de las letras que conforman la palabra. La asta y el travesaño de la $t$ se hicieron a la misma altura por lo que prácticamente son iguales. El parecido solo se interrumpe por la diferencia en la cantidad de picos en la $\mathrm{m}$. Mientras que en la carta Herrera traza tres picos, en la anotación del manuscrito solo escribe dos. Esta variante en las anotaciones al margen no deslegitima la atribución a la mano de Herrera pues dentro de todo el manuscrito se muestran varios errores de escritura ${ }^{36}$.

Dentro de la clasificación de Blecua, la variación en la cantidad de picos de la m puede considerarse un error de omisión, «el copista omite una letra, sílaba, palabra o frase de extensión variable cuando el elemento siguiente comienza o termina de forma igual o muy semejante» ${ }^{37}$. La repetición de los picos en la grafía de $m$ hace posible que se omita o duplique la cantidad. Estos descuidos de Herrera nos revelan dos aspectos importantes del manuscrito M. Por un lado, que fue escrito con celeridad y sin guardar mucho cuidado en la caligrafía; por otro, que su autor volvió a él en repetidas ocasiones para anotar rápidamente breves resúmenes del contenido. Este procedimiento se comprueba en una de las anotaciones al margen que repite la información registrada en el cuerpo del texto [imagen 9] ${ }^{38}$.

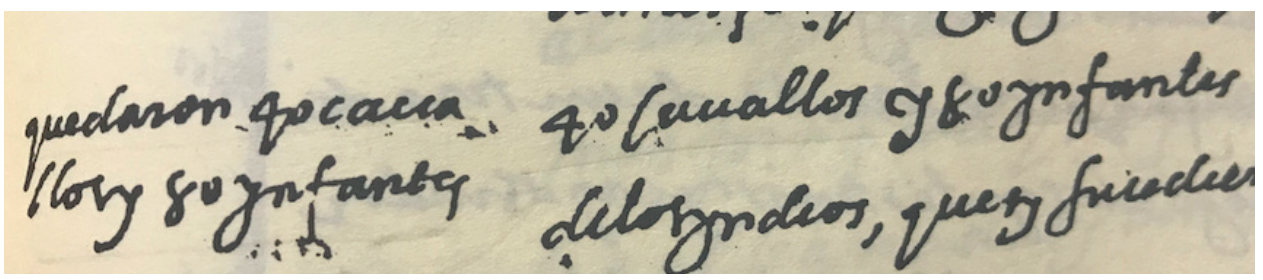

Imagen 9

En el cuerpo del manuscrito (a la derecha) se lee «40 cavallos y 80 ynfantes». A la izquierda, la anotación al margen repite los datos «quedaron 40 cavallos y 80 ynfantes». La similitud de las grafías es indiscutible. Los números también guardan extrema similitud con los años anotados en otras páginas en los márgenes del manuscrito [imagen 10 y 11$]^{39}$.

36. Para la clasificación y el estudio de estos errores seguiré el modelo propuesto por Alberto Blecua en su Manual de crítica textual. Basándose en las categorías modificativas aristotélicas, Blecua distingue cuatro tipos de errores que inconscientemente puede cometer un copista: «a) por adición (adiectio); b) omisión (detractadlo); c) alteración del orden (transmutatio), y d) por sustitución (immutatio)» (Blecua, 1983, pp. 19-20)

37. Blecua, 1983, p. 21

38. Maticorena Estrada, 2015, p. 209.

39. Maticorena Estrada, 2015, pp. 188 y 214 


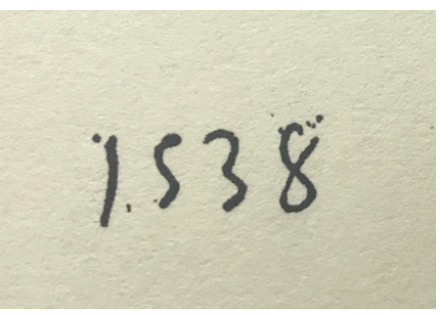

Imagen 10

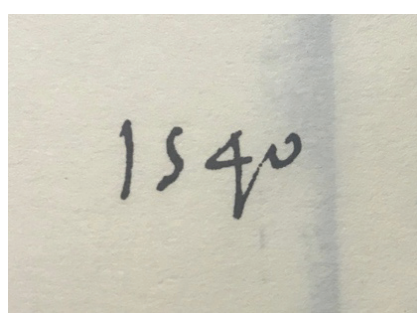

Imagen 11

Los trazos que componen el número ocho [imagen 9] coinciden con el escrito en el año 1538 [imagen 10]; así como el cuarenta [imagen 9] se asemeja al del año 1540 [imagen 11]. El ocho, en ambos casos, se compone de un solo trazo que no cierra en el ojal superior del número. El cuarenta presenta un enlace entre el cuatro y el cero en ambos casos. Asimismo, esta coincidencia se complementa con las grafías de una de las fechas en las cartas de Herrera «18 de octubre de 1605» [imagen 12].

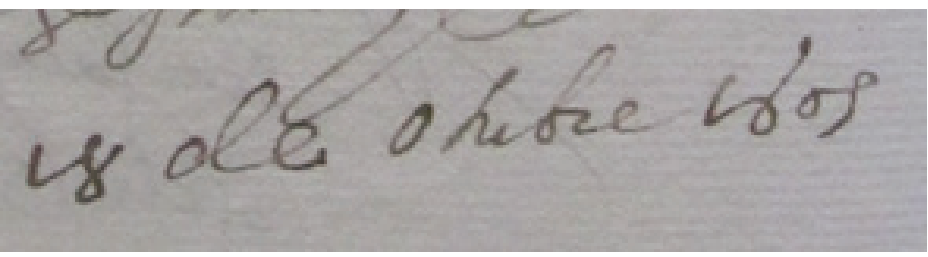

Imagen 12

El trazo del número ocho presenta las mismas características que las anotaciones marginales. Además, el cinco en la fecha de la carta también presenta una gran similitud con la anotación al margen pues se compone de un solo trazo erguido. Finalmente, para mayor comprobación de que la caligrafía presente en las diferentes partes del manuscrito es la misma, presento una comparación de la palabra flechas dentro de las anotaciones al final del texto [imagen 13] y en el cuerpo del texto [imagen14] ${ }^{40}$.

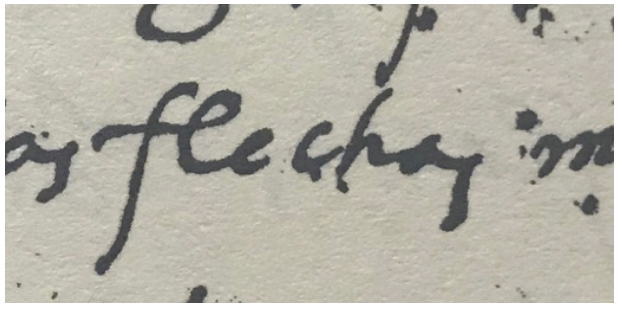

Imagen 13

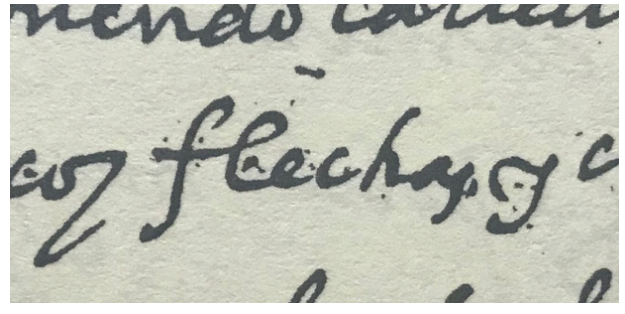

Imagen 14

40. Maticorena Estrada, 2015, p. 263 y 212. 
La composición de la palabra exhibe el mismo patrón: dos trazos para componer la $f$, uno para la asta y otro para el travesaño del cual se extiende el brazo que dibuja la I. La grafía de la $h$ se inclina hacia la derecha y de su base se recoge el trazo para la a, la composición de la s final es descendente y erguida.

Este cotejo es suficiente para comprobar que el cuerpo del manuscrito, así como las anotaciones al margen y las notas al principio y final del manuscrito, fueron creadas por la misma mano. La coincidencia de esta caligrafía con la correspondencia personal del cronista mayor de Indias comprueba que Antonio de Herrera y Tordesillas fue el autor, copista del original de Garcilaso, y amanuense del documento M. Tras asentar la identidad formal de la mano de Herrera en la ejecución de M es preciso pasar al análisis del contenido. En las siguientes páginas estudiaré algunos de los errores presentes en $\mathrm{M}$ que revelan el proceso de síntesis y alteración de la narración de La Florida que realizó Herrera.

\section{LA NATURALEZA DE LAS ENMIENDAS DE ANTONIO DE HERRERA}

Las últimas líneas del manuscrito $\mathrm{M}$ describen con un tinte trágico el retorno de los hombres de Hernando de Soto a la Habana. Allí los recibió la viuda de De Soto, Isabel de Bobadilla, quien después de enterarse del fatídico final de la expedición «murió poco después angustiada por la pérdida de su marido» ${ }^{41}$. Este final excluye el último capítulo de La Florida del Inca, en el que Garcilaso hace una relación de los misioneros fallecidos en La Florida. Sin embargo, la última página del manuscrito M contiene siete anotaciones entre las que se incluye un dato extraído del último capítulo de Garcilaso. La primera anotación señala que en 1549 el dominico fray Luis Cancel de Balvastro murió asesinado por los nativos. Este es el único dato del último capítulo de La Florida del Inca.

Las siguientes anotaciones se refieren a Garcilaso como autor y a un experimento realizado por los españoles para probar la resistencia de las cotas de maIla españolas contra las flechas de los indios. Ya que las cotas son atravesadas sin problemas por las flechas de los nativos, Herrera sugiere su reemplazo por los escaupiles, un tipo de túnicas acolchadas de algodón de origen mesoamericano que los españoles adoptaron para su defensa: «la prueba de las cotas que pasaban las flechas dice Garcilaso a hojas 192 [...] los escaupiles de lienzo estofados de algodón cuatro dedos en grueso con faldamientos largos f. 193 que en efecto son sayos para la defensa de las flechas mejores que las cotas de malla» ${ }^{42}$. Esto comprueba que Herrera tenía en su poder la versión final de la crónica de Garcilaso cuando elaboró el manuscrito M. Sobre el proceso de redacción del manuscrito,

41. Maticorena Estrada, 2015, p. 263.

42. Maticorena Estrada, 2015, p. 263. El pasaje referido por Herrera en la crónica de Garcilaso describe la fabricación y la forma de las túnicas de defensa. Sin embargo, Garcilaso no llama escaupiles a estas prendas. El término escaupil es de origen náhuatl y proviene de la unión de las palabras ichcatl 'algodón' y huipilli 'camisa' (ver la definición de escaupil que da la Real Academia Española en el Diccionario de la lengua española). En la primera parte de sus Décadas, Herrera describe la exploración de Hernán Cortés sobre Tezcoco; por lo cual, posiblemente, estaba familiarizado con algunos términos náhuatl. 
Maticorena indica que el escritor «parece que tiene a la vista otro manuscrito que no conocemos» ${ }^{43}$. Efectivamente, Herrera se encontraba mirando la versión inédita de La Florida del Inca mientras la resumía en M.

Las anotaciones finales de $\mathrm{M}$ reflejan dos aspectos centrales de la labor historiográfica de Herrera. Por un lado, dan preferencia a los temas militares, las armas y las técnicas de defensa de los españoles. Por otro lado, concentran la narración en la labor de los españoles. Mientras que Garcilaso presenta constantemente retratos ejemplarizantes entre nativos y españoles, Herrera suprime sistemáticamente los registros que describen la estructura social y las costumbres de los nativos de La Florida.

En el citado episodio de las cotas de malla, los españoles colocan dos cotas juntas y le piden a un prisionero indígena que lance una flecha contra ellas. La versión de Garcilaso señala que después de atravesar las cotas, el nativo de La Florida les pide a los españoles otro intento alegando que su habilidad podía superar al tiro anterior. Sin embargo, las versiones de $\mathrm{M}$ y las Décadas, que son casi idénticas, suprimen el diálogo y se concentran en la fiereza de los nativos. Dice M: «tan fieros son aquellos tiros y los indios tan diestros que se vio poner cuatro dobleces de malla en un cesto y tirar a prueba un indio y pasallo de claro por donde dejaron las finas cotas y usaron los escaupiles» ${ }^{44}$.

Los diálogos entre españoles y nativos tienen una función central en la crónica de Garcilaso ya que constantemente desafían la línea divisoria entre el barbarismo y la civilidad. La admiración que los nativos causan entre los españoles produce un retrato ambiguo donde se pierde de vista el orden jerárquico entre barbarismo y civilidad. Lisa Voigt señala que la ambigüedad dota de un giro positivo a la hostilidad de los nativos «as Garcilaso's ambiguous portrait of indigenous hostility toward Spaniards throughout La Florida, the Amerindian's threat might be interpreted as either insolent barbarism or laudable bravery» ${ }^{45}$. Garcilaso emplea la ambigüedad como un arma retórica que inserta a los nativos de la Florida en categorías medulares de la sociedad española como el honor, el valor militar y el servicio al bien común. Herrera altera sistemáticamente la mayoría de estos cuadros ambiguos, aunque no siempre logra borrar completamente las huellas de la retórica de Garcilaso.

Sobre las alteraciones intencionales, Blecua afirma: «Todo error supone un cambio, pero no todo cambio supone un error» ${ }^{46}$. Así, las enmiendas de $\mathrm{M}$ se pueden clasificar en dos grupos. Primero, se encuentran los errores típicos de un copista. Entre ellos están la omisión y la alteración del orden de los grafemas en palabras y en nombres propios. Segundo, están las enmiendas que revelan el proceso constante de síntesis y recomposición de la información que Herrera realizó a partir de la versión inédita de La Florida del Inca. Estas enmiendas demuestran que Herrera releyó e intervino la información del manuscrito $\mathrm{M}$ en varias ocasiones. 
Los dos tipos de enmiendas están diseminados a lo largo del manuscrito M. No obstante, algunos episodios se distinguen por presentar mayor cantidad de modificaciones. Uno de ellos es el encuentro entre Juan Ortiz, un sobreviviente de la expedición de Pánfilo de Narváez, y los hombres de Hernando de Soto. La Florida del Inca describe que Juan Ortiz había sido prisionero del líder Hirrihigua, quien solía torturarlo en venganza por los maltratos recibidos de Narváez. Con la ayuda de una de las hijas de Hirrihigua, Ortiz escapa y se refugia con el joven líder Mucozo quien lo protege y ayuda a reunirse con los españoles. Garcilaso afirma que Ortiz pasó diez años viviendo entre los indios de la Florida, «el uno y medio en poder de Hirrihigua y los demás con el buen Mucozo» antes de encontrarse con el ejército de De Soto ${ }^{47}$.

El manuscrito $\mathrm{M}$ contiene una tachadura y enmienda en la sección que refiere los años del cautiverio de Ortiz: «después de [Tachado: algunos años] que estuvo en su poder» ${ }^{48}$. Herrera coloca el dato «año y medio» sobre la tachadura. Es decir, luego de releer esta sección, decidió conservar el dato y por eso lo colocó sobre la tachadura. Esta enmienda es una muestra de las múltiples ediciones que Herrera realizó sobre el manuscrito M después de su primera redacción.

Las tachaduras siguientes responden a otro propósito: «[Tachado: una] por [Tachado: su res] respeto dellos le amparó otro cacique llamado Mucozo» ${ }^{49}$. Estas tachaduras corresponden a la labor de síntesis de información durante la primera redacción del manuscrito. Por esta razón, la corrección continúa dentro del texto y no se ubica arriba de la tachadura. Al mismo propósito responde la tachadura siguiente: «[Tachado: que no llevaban más] desnudos» ${ }^{0}$. Herrera no demuestra mayor interés por los datos de tipo etnográfico, así que suprime varias descripciones del arte indígena como la vestimenta.

A pesar de estas ediciones, Herrera no borra por completo los espacios de ambigüedad creados por Garcilaso. Antes de reunirse con los españoles, Garcilaso describe que los nativos aconsejan a Ortiz que actúe con prudencia hasta encontrarse fuera de peligro.

Los indios, viendo los castellanos, dijeron a Juan Ortiz que sería cordura asegurar sus personas y vidas con meterse en el monte hasta que los cristianos los reconociesen por amigos [...]. Juan Ortiz no quiso tomar el buen consejo de los indios, confiado en que era español y que los suyos le habían de conocer luego que le viesen, como si viniera vestido a la española o estuviera en alguna cosa diferenciado de los indios para ser conocido por español ${ }^{51}$.

El encuentro produce una confusión que casi le cuesta la vida a Ortiz y a un indio que no logró escapar a tiempo de los españoles. Los episodios donde se confunde como indio a un español sobreviviente son comunes en varias crónicas coloniales.

47. Inca Garcilaso de la Vega, La Florida del Inca, p. 54

48. Maticorena Estrada, 2015, p. 190.

49. Maticorena Estrada, 2015, p. 190.

50. Maticorena Estrada, 2015, p. 191.

51. Inca Garcilaso de la Vega, La Florida del Inca, p. 58 
Estos se caracterizan por exponer la dimensión engañosa de la apariencia. Jerónimo de Aguilar en La historia verdadera de la conquista de la Nueva España (1632) de Bernal Díaz del Castillo o Alvar Núñez Cabeza de Vaca en sus Naufragios (1542) son algunos casos representativos.

Garcilaso eleva estos episodios hacia un nivel más complejo. En la primera parte de los Comentarios reales, el episodio del náufrago Pedro Serrano no solo expone la trampa de las apariencias, sino que anula las jerarquías entre los dos protagonistas del encuentro, ya que ambos son españoles creyendo que el otro es el demonio ${ }^{52}$. En el caso de Juan Ortiz, la inversión también sobrepasa el ámbito de las apariencias y se adentra en las facultades del entendimiento. Los nativos que aconsejan a Ortiz que se esconda demuestran un comportamiento prudente, una facultad del tópico medieval sapientia et fortitudo ${ }^{53}$ que se aplicaba a los héroes épicos. Por ello, luego de aclarada la confusión, Garcilaso afirma que los nativos reprendieron a Ortiz «llamándole tonto, necio, impertinente, que no era español ni hombre de guerra» ${ }^{54}$. Este primer encuentro con los nativos adelanta la flexibilidad con que Garcilaso va a manejar las jerarquías de barbarie y civilidad entre nativos y españoles.

En el manuscrito $\mathrm{M}$, a pesar de las supresiones, la ambigüedad entre las jerarquías no se pierde por completo. Herrera sintetiza el episodio señalando que Ortiz «confiando más de lo que debiera no quiso tomar el buen consejo de los bárbaros» ${ }^{55}$. También incluye las reprimendas de los nativos. Sin embargo, no las describe: «le deshonraban con muchas injurias diciendo que por inadvertencia había sucedido aquella desgracia» ${ }^{56}$. Más tarde, estos nativos se vuelven aliados de Hernando de Soto, proveyéndole de recursos y permitiéndole avanzar sin obstáculos por su territorio. Luego, Garcilaso presenta a Mucozo, el líder de esta comunidad, como un ejemplo de príncipe. Las alianzas entre los líderes nativos y los españoles fueron muy convenientes para legitimar la carrera imperial de España. Probablemente, este aspecto de La Florida de Garcilaso fue determinante para que Herrera decida insertarla en la crónica oficial.

Las enmiendas de este episodio también demuestran que el manuscrito M se compuso a partir de la versión inédita pero completa de La Florida del Inca que incorpora todas las fuentes de información a las que accedió Garcilaso. La primera versión de La Florida del Inca fue escrita a partir del relato oral de Gonzalo Silvestre.

\footnotetext{
52. Varios investigadores han estudiado el episodio de Pedro Serrano como una metáfora viva que Garcilaso emplea para problematizar de la figura del salvaje. Ver Firbas, 2004; Guerra, 2009; Ledezma, 2010. 53. El tópico medieval sapientia et fortitudo combina las virtudes morales y físicas del héroe-guerrero. La fortaleza física que distingue al guerrero épico y le facilita la victoria en batalla componen el aspecto de fortituto. En contraparte, la mesura que lo distingue como un modelo de conducta para la vida cotidiana está considerado en la sapientia. Norman Schafler identifica al Cid Campeador como el máximo modelo de este tópico: «these two attributes complement one another in the Cid's make-up, and it is from these two that the other ideals the critics have seen embodied in the character of Rodrigo are derived» (1977, p. 44).

54. Inca Garcilaso de la Vega, La Florida del Inca, p. 60.

55. Maticorena Estrada, 2015, p. 191

56. Maticorena Estrada, 2015, p. 191
} 
Posteriormente, Garcilaso accedió a las crónicas de Alonso de Carmona y Juan Coles, con las que reescribió su historia. En el episodio de Juan Ortiz, Garcilaso distingue la información que toma de Coles de la siguiente manera:

[Juan Ortiz] viendo que Álvaro Nieto revolvía sobre él, dio grandes voces diciendo «Xivilla, Xivilla», por decir Sevilla, Sevilla. En este paso, añade Juan Coles que, no acertando Juan Ortiz a hablar castellano, hizo con la mano y el arco la señal de la cruz para que el español viese que era cristiano. Porque, con el poco o ningún uso que entre los indios había tenido de la lengua castellana se le había olvidado pronunciar hasta el nombre de la propia tierra ${ }^{57}$.

Garcilaso complementa la versión de Silvestre con la de Coles. En el manuscrito $\mathrm{M}$, Herrera sintetiza ambas versiones. La narración combina las exclamaciones «Xivilla» de Silvestre, con el paso sobre la señal de la cruz de Coles:

[...] Joan Ortiz contra el que arremetió un soldado llamado Álvaro Nieto, natural de Albuquerque, y rebatiendo con el arco el golpe de la lanza dio un salto atrás y dijo Xibilla Xibilla porque había olvidado la lengua natural y con el arco hizo la señal de la cruz [Tachado: tomole] preguntole Nieto si era [Tachado: llamaba Juan] el Juan Ortiz; dijo que sí; tomole en las ancas del caballo58.

La versión final de Herrera descarta varios elementos de M, reduce la información y guarda el sentido general del episodio. En el caso del episodio de Juan Ortiz, Herrera omite la fuente principal de Garcilaso y conserva el añadido de Juan Coles. Teniendo en cuenta que solo el Inca tuvo acceso a estas fuentes secundarias, se infiere que el manuscrito M sintetiza los contenidos de una versión completa y final de La Florida del Inca.

\section{CONCLUSIONES}

De los argumentos expuestos se pueden extraer algunas conclusiones importantes para el posterior estudio tanto de La Florida de Garcilaso como de las Décadas de Herrera. Queda comprobado que Antonio de Herrera desarrolló un vivo interés por la obra del Inca desde que las prerrogativas de su cargo le permitieron el acceso al manuscrito inédito de La Florida del Inca. Por esta razón el cronista mayor agregó el nombre del Inca al índice de autores consultados en el primer tomo de sus Décadas publicado en 1601, a pesar de que esta primera entrega no cubría la expedición de Hernando de Soto. La presencia del nombre de Garcilaso en el índex de Herrera comprueba que el manuscrito inédito de La Florida llegó a manos del cronista mayor poco después de haber sido remitido al Consejo de Indias para su evaluación.

Es también probable que Herrera haya utilizado la prueba de impresión de La Florida numerada y revisada por el escribano del Consejo como base para escribir M. Según el procedimiento establecido, una vez cotejada la prueba de impresión

57. Inca Garcilaso de la Vega, La Florida del Inca, pp. 58-59. Cursivas mías.

58. Maticorena Estrada, 2015, p. 191. 
con el manuscrito original se otorgaba la licencia de publicación al autor junto con la tasa y el privilegio. Garcilaso efectivamente obtuvo la licencia de publicación para La Florida a finales de mayo de 159959. Sin embargo, la impresión no se realizó. Cabe la posibilidad de que Herrera haya retenido esta prueba de impresión de La Florida para elaborar M; por lo cual, a pesar de contar con la licencia, Garcilaso no logró publicar su obra.

El cotejo de la caligrafía de M con el epistolario personal de Antonio de Herrera comprueba que el cronista mayor es el autor del documento encontrado por Maticorena. La identificación de Herrera como el autor autógrafo del manuscrito M permite el análisis de los criterios de edición aplicados sobre La Florida del Inca. Estos criterios responden principalmente a dos objetivos. Por un lado, Herrera borra sistemáticamente las huellas de la retórica de Garcilaso para así desligar la narración del debate sobre las facultades de los nativos de La Florida. Mientras que Garcilaso emplea la ambigüedad para construir una representación favorable de los líderes nativos, Herrera se enfoca en presentarlos como colaboradores y potenciales aliados de los españoles. Por otro lado, el cronista mayor discrimina entre los datos y escoge aquellos que considera que reforzarán la verosimilitud de la obra y la empresa de la conquista de La Florida.

En líneas generales, tanto Garcilaso como Herrera compartieron el mismo interés por estimular la exploración y conquista de la Florida. Mediante el estudio de las variantes del manuscrito M y las Décadas de Herrera, se aclaran los propósitos políticos por los cuales Antonio de Herrera privilegió la obra de Garcilaso para escribir la crónica oficial española. El tratamiento que Herrera le da a la obra de Garcilaso nos permite comprender mejor los capítulos más tempranos de la historia de la conquista de La Florida española.

\section{BiBLIOGRAFÍA}

Adorno, Rolena, «"Obedezco pero no cumplo": Surviving Censorship in Early Modern Spain», Textual Cultures, 13, 2020, pp. 29-74.

Asensio, Eugenio, «Dos cartas desconocidas del Inca Garcilaso», Nueva Revista de Filología Hispánica, 3.4, 1953, pp. 583-593.

«Aguado, Pedro de», en Real Academia de la Historia, Diccionario Biográfico Electrónico, 2020 [consulta: 03/03/2020].

Blecua, Alberto, Manual de crítica textual, Madrid, Castalia, 1983.

Dewhurst, William, The History of Saint Augustine, Florida, New York, Rutland, 1968.

59. Pedro Guibovich (2006, p. 201) señala que, al contrario de lo que afirmaba José Durand, a fines de mayo de 1599 Garcilaso obtuvo la licencia de publicación, por lo cual cedió a Juan de Morales los derechos de impresión y venta de La Florida con el afán de ver impresa su obra. Así lo confirma un poder firmado por el Inca Garcilaso y Gonzalo Fernández de Córdoba, escribano público de Córdoba, el 29 de mayo de 1599. Ver de la Torre y del Cerro, 1935, pp. 60-62. 
Durand, José, «La redacción de La Florida del Inca: cronología», Revista Histórica, XXI, 1954, pp. 283-302.

Durand, José, «Garcilaso Inca jura decir verdad», Histórica, XIV, 1990, pp. 1-25.

Elvas, Fidalgo de, Relação verdadeira dos trabalhos que o governador D. Fernando de Souto e certos fidalgos portugueses passaram no descobrimento da província da Florida, agora novamente feita por um fidalgo de Elvas, ed. Maria da Graça Mateus Ventura, Lisboa, Comissão Nacional para as Comemorações dos Descobrimentos Portugueses, 1998.

Firbas, Paul, «La geografía antártica y el nombre del Perú», en La formación de la cultura virreinal, ed. Karl Kohut, Madrid / Frankfurt am Main, Iberoamericana / Vervuert, 2004.

Garcilaso de la Vega, Inca, Comentarios reales de los Incas, Lima, Banco de Crédito del Perú, 1985.

Garcilaso de la Vega, Inca, La Florida del Inca, México, Fondo de Cultura Económica, 1956.

Garcilaso de la Vega, Inca, Relación de la descendencia del famoso Garcí-Pérez de Vargas con algunos pasos de historias dignas de memoria [consulta: 03/06/2020].

Guerra, Estrella, «La digresión en los Comentarios reales de los Incas: ¿oportunidad para la ficción? El caso de Pedro Serrano», Lexis, XXXIII, 2009, pp. 325-339.

Guibovich Pérez, Pedro, «La publicación de La Florida del Inca y su contexto histórico: problemas y perspectivas de investigación», en Franqueando fronteras. Garcilaso de la Vega y "La Florida del Inca», ed. Raquel Chang-Rodríguez, Lima, Pontificia Universidad Católica del Perú, 2006, pp. 201-211.

Herrera y Tordesillas, Antonio de, Historia general de los hechos de los castellanos en las islas de Tierra Firme y del mar océano, Madrid, Imprenta Real, 1601.

Hoffman, Paul, The Spanish Crown and the Defense of the Caribbean, 1535-1585: Present, Patrimonialism, and Royal Parsimony, Louisiana, Louisiana State University Press, 1999.

Hopkins, Eduardo, «Prólogo», en La Florida: facsímil de un nuevo manuscrito, Lima, Fondo Editorial de la Universidad Ricardo Palma, 2015, pp. 13-21.

Ledezma, Domingo, «Los infortunios de Pedro Serrano: huellas historiográficas de un relato de naufragio», Renacimiento mestizo: los 400 años de los "Comentarios reales», ed. José Antonio Mazzotti, Iberoamericana, 2010, pp. 30-46.

«Madrigal, Pedro», en Real Academia de la Historia, Diccionario Biográfico Electrónico, 2020 [consulta: 01/04/2020].

Martínez Torrejón, José Miguel, Los pre-textos de «La Florida del Inca», Alicante, Universitat d'Alacant, 2020. 
Maticorena Estrada, Miguel, «La Florida»: facsímil de un nuevo manuscrito, Lima, Fondo Editorial de la Universidad Ricardo Palma, 2015.

Mc Grath, John, The French in Early Florida, Gainesville, University Press of Florida, 2000.

Miró-Quesada, Aurelio, «Prólogo», en La Florida del Inca, México, FCE, 1956, pp. IX-LXXVI.

Pérez Pastor, Cristóbal, La imprenta en Medina del Campo, Madrid, Sucesores de Rivadeneyra, 1895.

Real Academia Española, Diccionario de la lengua española, 2020 [consulta: 20/06/2020].

Riva-Agüero, José de la, Estudios de historia peruana. La historia del Perú, Lima, PUCP, 1965

Schafler, Norman, «"Sapientia et fortitude" in the Poema de mío Cid», Hispania, 60, 1977, pp. 44-50.

Tebeau, Charles, A History of Florida, Miami, University of Miami Press, 1971.

Torre Revello, José, El libro, la imprenta y el periodismo en América durante la dominación española, Buenos Aires, Casa Jacobo Peuser, 1940.

Torre y del Cerro, José de la, El Inca Garcilaso de la Vega (nueva documentación), Sevilla, Instituto Hispano-cubano de historia de América, 2012.

Voigt, Lisa, Writing Captivity in the Early Modern Atlantic. Circulations of Knowledge and Authority in the Iberian and English Imperial Worlds, Chapel Hill, University of North Caroline Press, 2009. 